\title{
REALISTIC MEASUREMENT OF PHASE
}

\author{
H. Paul and U. Leonhardt \\ Arbeitsgruppe "Nichtklassische Strahlung" der Max-Planck-Gesellschaft \\ an der Humboldt-Universität Berlin, Rudower Chaussee. 5, 12484 Berlin, Germany
}

The experimental schemes for measuring quantum-mechanical phase properties of light suggested and partly also realized thus far, namely (i) amplification, with the help of a quantum amplifier, of the microscopic field before phase measurement, (ii) heterodyning the field with a strong local oscillator, and (iii) performing two separate homodyne measurements on the field after beam splitting, are compared from a theoretical point of view. They share the common feature that undesired noise enters the experimental setup, which makes the measurement fuzzy. It will be pointed out that all three schemes amount to measuring the $Q$ function of the original field, and hence are fully equivalent. Since the $Q$ function can be interpreted as a smoothed Wigner function, one may associate with the introduced noise a smoothing process in which intrinsically quantum-mechanical features displayed by finer details of the Wigner function - especially by the occurrence of negative values - are lost. As a consequence, the measured phase distribution will be broader than the "true" one based on the concept of a quantum-mechanical phase operator. In realistic experiments, the nonunit detection efficiency further deteriorates the measuring results. It will be shown that also this effect can be properly described by an (additional) smoothing process leading to a certain $s$-parametrized quasiprobability distribution, with a parameter $s$ that is connected with the detection efficiency in a simple way, as the distribution that is actually measurable.

PACS numbers: $42.50 . \mathrm{Wm}, 03.65 . \mathrm{Bz}$

\section{Introduction}

The quantum-mechanical description of phase by introducing a Hermitian phase operator [1] suffers, apart from the fact that this problem could be solved in a perfectly satisfactory way only by resorting to a finite-dimensional Hilbert space [2], from the lack of any prescription for an actual phase measurement, even in form of a Gedanken experiment. Therefore, in order to make contact with reality, one will have to turn the tables. One will first devise an experimental scheme for measuring phase properties, thus giving an operational definition of phase, and afterwards search for the proper quantum-mechanical description of the experimental procedure. In fact, several schemes for phase measurement which 
differ distinctly in their experimental setup have already been proposed and even partly realized. We will describe them in some detail and afterwards present the outlines of their theoretical analysis.

\section{Measuring schemes}

The first to discuss a realistic phase measuring device were Bandilla and Paul [3], who as early as 1969 proposed to amplify, with the help of a laser (or parametric) amplifier, the microscopic field to be investigated to a macroscopic level, where classical phase measurement techniques can readily be applied. Since any amplifier unavoidably adds noise to the amplified signal, this type of phase measurement is necessarily of noisy (fuzzy) character.

Fifteen years later Shapiro and Wagner [4] analyzed a heterodyne detection scheme which allows simultaneous, however noisy, observations of both the phase and the amplitude of a signal field. Their basic idea was to mix the signal, by means of a weakly reflecting mirror, with a strong coherent field (local oscillator) whose frequency is shifted by a certain amount $\Delta \nu$ (see Fig. 1). The mixed field is sent to

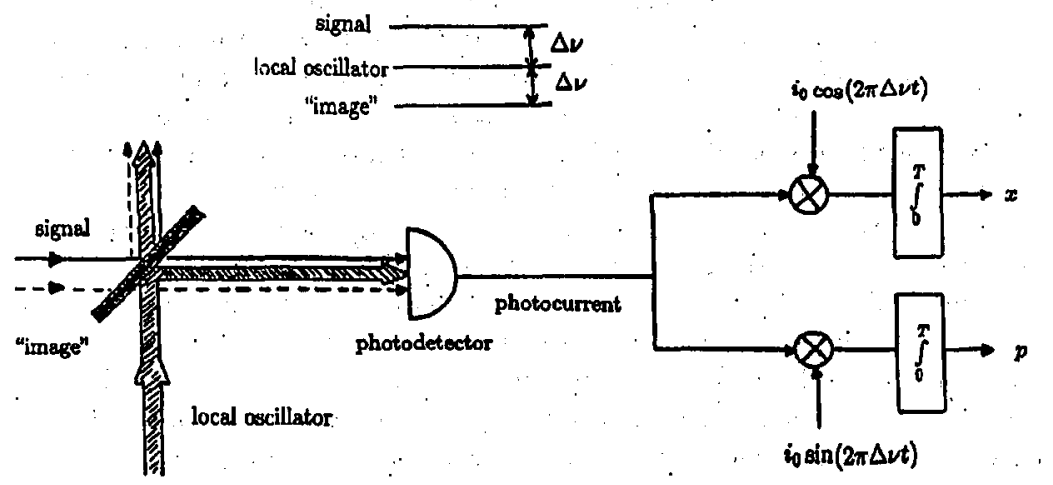

Fig. 1. Heterodyne detection scheme that allows to perform a simultaneous, however noisy, measurement of the two quadrature components of the field.

a photodetector. Its photocurrent contains an alternating current oscillating at the difference frequency $\Delta \nu$ - it is just the beat signal - and the amplitudes of this alternating current, corresponding to the components oscillating as $\cos (2 \pi \Delta \nu t)$ and $\sin (2 \pi \Delta \nu t)$, respectively, are determined separately by well-known electronic techniques (see Fig. 1). By repeating this measurement many times, one can determine a distribution function for those amplitudes $x$ and $p$. Passing to polar coordinates one gets a distribution function for both the amplitude (radius) and the phase (polar angle) of the signal field. Averaging, in particular, over the amplitude yields a phase distribution (cf. Sec. 3). It must be emphasized that also in the present case undesired noise enters the experimental device. One has to notice that a beat signal with beat frequency. $\Delta \nu$ originates also from the field mode 
which is the image, with respect to frequency, of the signal mode (see Fig. 1), even when this mode is empty. In this case vacuum fluctuations are still present which get mixed with the local oscillator and thus make the measurement noisy.

Only recently, Noh, Fougères and Mandel [5] (cf. also [6]) proposed and, moreover, realized a different experimental scheme which is closely related to classical phase measurement. Hence its basic features can easily be understood. Let us stress that even in classical optics there exists no device which allows to determine the phase in a single measurement. The deeper reason for this is that radiation interacts with matter only via the electric field strength in which phase and amplitude are combined so that any single measurement yields a complex information on both phase and amplitude. Hence, at least two different measurements are needed to determine the phase and the amplitude separately. A simple way to do this is suggested by writing the electric field strength as a sum of two components that are in phase and out of phase, respectively, compared to a given reference field

$$
E(r, t)=E_{0} \cos (k r-\omega t-\varphi)=C[x \cos (k r-\omega t)+p \sin (k r-\omega t)] .
$$

Here, $x$ and $p$ are the so-called quadrature components of the field and $C$ is a normalization constant. It is readily inferred from Eq. (1) that $\cos \varphi$ and $\sin \varphi$ can be expressed through $x$ and $p$ as

$$
\cos \varphi=\frac{x}{\sqrt{x^{2}+p^{2}}}, \quad \sin \varphi=\frac{p}{\sqrt{x^{2}+p^{2}}}
$$

Those formulas suggest the following scheme for phase measurement: measure simultaneously $x$ and $p$ and calculate $\cos \varphi$ and $\sin \varphi$ with the help of Eqs. (2). In practice, one will use a beam splitter and measure $x$ and $p$ separately on the two outgoing beams.

Now one might ask, why cannot we use a similar scheme for a quantum measurement of phase? At first sight, it scems impossible since $x$ and $p$ are actually variables that are canonically conjugate (they are similar to position and momentum of a particle which explains also our notation) and, hence, cannot be measured simultaneously on principle. But why should not we use a beam splitter and measure $x$ on one of the outgoing beams and $p$ on the other? The answer is, of course you can do this, but what you are performing in this way is a noisy measurement of $x$ and $p$. In fact, the beam splitter introduces additional noise since a vacuum field enters its unused input port. So restricted accuracy is the price one has to pay for a simultaneous measurement of canonically conjugate variables. The actual measurement of $x$ and $p$ can be done utilizing a balanced homodyne detection scheme (see Fig. 2). The signal is mixed with a reference field (local oscillator), and the two outgoing fields are made to shine on separate photodetectors. The difference of the two photocurrents gives us, up to a factor, the quadrature component $x$, and when one shifts the phase of the local oscillator by $\pi / 2$ (e.g. by inserting a quarter-wave plate), one measures $p$. Therefore, we arrive at the measuring scheme sketched in Fig. 3. We will assume that the reference beams are very strong, compared to the signal field, coherent fields so that the observed phase properties actually reflect features of the signal field not distorted by the reference fields. (It should be noted that in the actual experiments a weak local oscillator was used which makes the theoretical treatment rather involved [5].) 


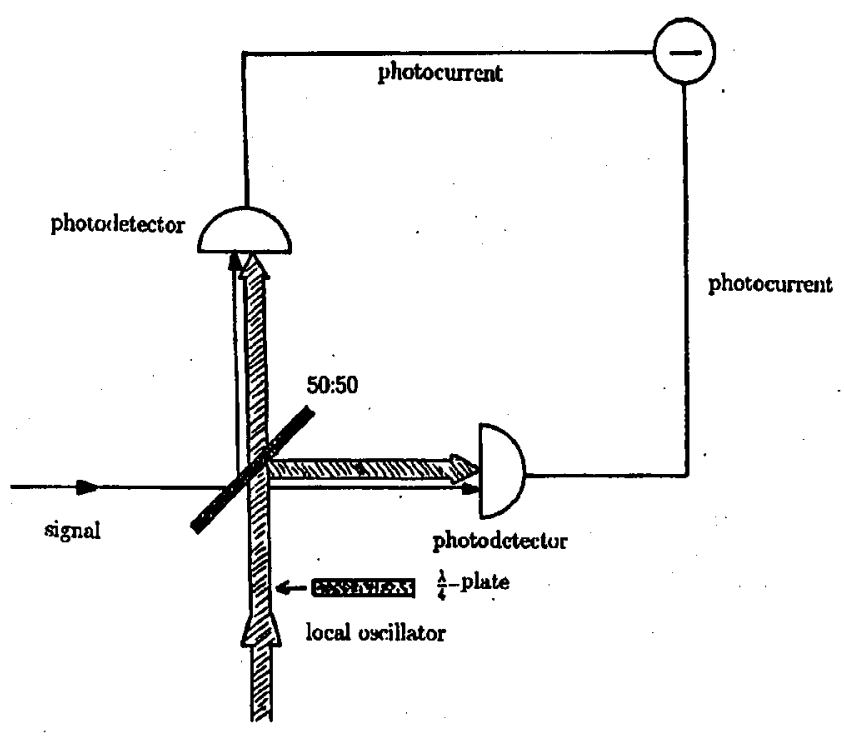

Fig. 2. Balanced homodyne detection scheme.

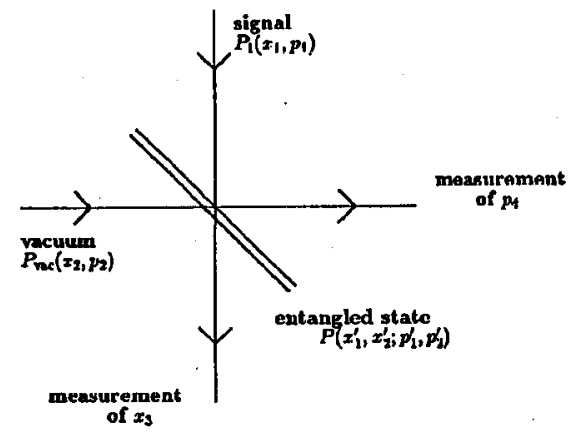

Fig. 3. Experimental scheme for a simultaneous, however noisy, measurement of the two quadrature components of the field via homodyne detection with strong coherent fields.

\section{Theory}

Let us now describe the aforementioned experiment in the quantum mechanical formalism (see Fig. 3). Since the description of the action of a (lossless) beam splitter takes its simplest form in the $x$ representation, we will use the latter [7].

It is advantageous to represent the state of the incident signal field by a Wigner function $P_{1}\left(x_{1}, p_{1}\right)$ [8]. The vacuum field entering the unused port of the beam splitter is characterized by the Wigner function

$$
P_{\mathrm{vac}}\left(x_{2}, p_{2}\right)=\pi^{-1} \exp \left[-\left(x_{2}^{2}+p_{2}^{2}\right)\right] \text {. }
$$

The action of a beam splitter with transmittivity $\cos ^{2} \Theta$ is simply described by a 
rotation in the $x_{1}, x_{2}-$ and the $p_{1}, p_{2}-$ plane [9]

$$
\left(\begin{array}{l}
x_{1} \\
x_{2}
\end{array}\right)=\left(\begin{array}{cc}
\cos \Theta & \sin \Theta \\
-\sin \Theta & \cos \Theta
\end{array}\right)\left(\begin{array}{l}
x_{1}^{\prime} \\
x_{2}^{\prime}
\end{array}\right), \quad\left(\begin{array}{l}
p_{1} \\
p_{2}
\end{array}\right)=\left(\begin{array}{cc}
\cos \Theta & \sin \Theta \\
-\sin \Theta & \cos \Theta
\end{array}\right)\left(\begin{array}{l}
p_{1}^{\prime} \\
p_{2}^{\prime}
\end{array}\right)
$$

where $x_{1}^{\prime}, p_{1}^{\prime}$ and $x_{2}^{\prime}, p_{2}^{\prime}$ are the quadrature components for the two outgoing bcams. For the special case of a 50:50 beam splitter $(\Theta=\pi / 4)$ Eqs. (4) reduce to

$$
\left(\begin{array}{l}
x_{1} \\
x_{2}
\end{array}\right)=2^{-\frac{1}{2}}\left(\begin{array}{cc}
1 & 1 \\
-1 & 1
\end{array}\right)\left(\begin{array}{l}
x_{1}^{\prime} \\
x_{2}^{\prime}
\end{array}\right), \quad\left(\begin{array}{l}
p_{1} \\
p_{2}
\end{array}\right)=2^{-\frac{1}{2}}\left(\begin{array}{cc}
1 & 1 \\
-1 & 1
\end{array}\right)\left(\begin{array}{l}
p_{1}^{\prime} \\
p_{2}^{\prime}
\end{array}\right) .
$$

Hence the Wigner function of the total system (before detection) is given by

$$
P_{\text {out }}\left(x_{1}^{\prime}, x_{2}^{\prime} ; p_{1}^{\prime}, p_{2}^{\prime}\right)=P_{1}\left(x_{1}, p_{1}\right) P_{\text {vac }}\left(x_{2}, p_{2}\right) \text {. }
$$

Here, the variables $x_{1}, p_{1}, x_{2}, p_{2}$ are to be substituted according to Eqs. (5). Assuming that $x_{1}^{\prime}$ and $p_{2}^{\prime}$ are measured, we have to integrate over the remaining variables $x_{2}^{\prime}$ and $p_{1}^{\prime}$ in order to get the probability distribution $w\left(x_{1}^{\prime}, p_{2}^{\prime}\right)$ for this simultaneous measurement. Considering the equations for $x_{1}$ and $p_{1}$ in (5) as substitutions, we may write the integral in question in the form of a convolution with a Gaussian

$$
\begin{aligned}
& w\left(x_{1}^{\prime}, p_{2}^{\prime}\right) \equiv \int P\left(x_{1}^{\prime}, x_{2}^{\prime} ; p_{1}^{\prime}, p_{2}^{\prime}\right) \mathrm{d} x_{2}^{\prime} \mathrm{d} p_{1}^{\prime} \\
& \quad=2 \pi^{-1} \int P_{1}\left(x_{1}, p_{1}\right) \exp \left[-\left(x_{1}-2^{\frac{1}{2}} x_{1}^{\prime}\right)^{2}-\left(p_{1}-2^{\frac{1}{2}} p_{2}^{\prime}\right)^{2}\right] \mathrm{d} x_{1} \mathrm{~d} p_{1} .
\end{aligned}
$$

Ilence the measured distribution originates from the original Wigner function by a certain smoothing process. The latter makes evident the noisy (fuzzy) character of the measurement that is due to the introduction of undesired noise. Actually, the convolution (7) is just the (appropriately scaled) $Q$ function for the incident field

$$
w\left(x_{1}^{\prime}, p_{2}^{\prime}\right)=2 Q\left(2^{\frac{1}{2}} x_{1}^{\prime}, 2^{\frac{1}{2}} p_{2}^{\prime}\right)
$$

The phase distribution is readily obtained from Eq. (8) as a marginal distribution, i.e. by introducing polar coordinates

$$
x_{1}^{\prime}=\varrho \cos \varphi, \quad p_{2}^{\prime}=\varrho \sin \varphi
$$

and averaging over the amplitude $\varrho$.

The smoothing process just described is actually associated with a certain loss of information. Finer details of the Wigner function become lost - in particular, negative values must disappear since the $Q$ function is positive definite. Hence the measured phase distribution will be broader than that following from the phase operator concept $[1,2]$.

From the formal point of view one might object that the $Q$ function contains precisely the same information as the Wigner function. This is true, however, only when the $Q$ function is known as an analytic function since only in that case the deconvolution can actually be performed.

Now, it is very interesting to note that both the amplification scheme proposed by Bandilla and Paul [3] and the heterodyne-detection scheme discussed by Shapiro and Wagner [4] have been shown to amount to measuring the $Q$ function of the incident field, too $[10,4]$. Therefore we can state that those two approaches 
are perfectly equivalent to Mandel's procedure [5]. In particular, they all lead to identical (measured) phase distributions, despite the significant differences in their experimental setup and the physical processes involved. It should be noticed, however, that they actually share the common feature that undesired additional noise is introduced either by beam splitting or by amplification.

At first sight, one might find it surprising that those different sources of noise should give rise to precisely the same enhancement of phase (as well as amplitude) fluctuations, compared to the "true" fluctuations present in the original field according to the phase operator concept [2]. It should be noted, however, that formally amplifier and beam splitter noise share a common property. Both of them enter, in the form of Langevin forces, the quantum mechanical equations of motion just in such a way as to preserve the quantum mechanical commutation relations for the photon creation and annihilation operators in the course of interaction, either with the beam splitter or the amplifying medium. From this argument, one may actually find the cquivalence of all operational definitions of phase discussed so far, rather quite natural.

\section{The influence of nonunit detection efficiency}

Thus far we have assumed that the observation is made with unit-efficiency detectors. However, such ideal detectors are not available in practice. (Note that the effective detection efficiency $\eta$ includes also losses, in particular those due to mode mismatch.) It is well known that low-efficiency detectors play a deteriorating role in studying quantum features. In the following, we want to give a quantitative account of this effect in Mandel's scheme [5] (see also Ref. [11]).

\subsection{Balanced homodyne detection}

As is well known [12], a realistic detector can be modelled by an ideal one $(\eta=1)$ with a partly transmitting mirror (a special form of an attenuator) in front of it. Ilence, in the homodyne detection schenie such a fictitious beam splitter has to be placed before each of the two detectors (see Fig. 4a). It is not difficult to show that this setup can actually be simplified [11]: one beam splitter attenuating the signal before it is mixed with the local oscillator has the same effect (see Fig. 4b).

\subsection{Beam splitling}

In the present case vacuum noise enters the setup not only via the beam splitter dividing the incident field, but also via the two fictive beam splitters placed before each homodyne-detection apparatus, according to the equivalent model introduced above (see Fig. 5). The theoretical description is a straightforward generalization of the procedure in Sec. 3. After some algebra one arrives at the following simple result for the measured distribution (for details see Ref. [11]):

$$
\begin{aligned}
& w\left(x_{1}^{\prime \prime}, p_{2}^{\prime \prime}\right)=2 \pi^{-1}(2-\eta)^{-1} \int P_{1}(x, p) \\
& \quad \times \exp \left\{-\frac{\eta}{2-\eta}\left[\left(x-2^{\frac{1}{2}} \eta^{-\frac{1}{2}} x_{1}^{\prime \prime}\right)^{2}+\left(p-2^{\frac{1}{2}} \eta^{-\frac{1}{2}} p_{2}^{\prime \prime}\right)^{2}\right]\right\} \mathrm{d} x \mathrm{~d} p .
\end{aligned}
$$



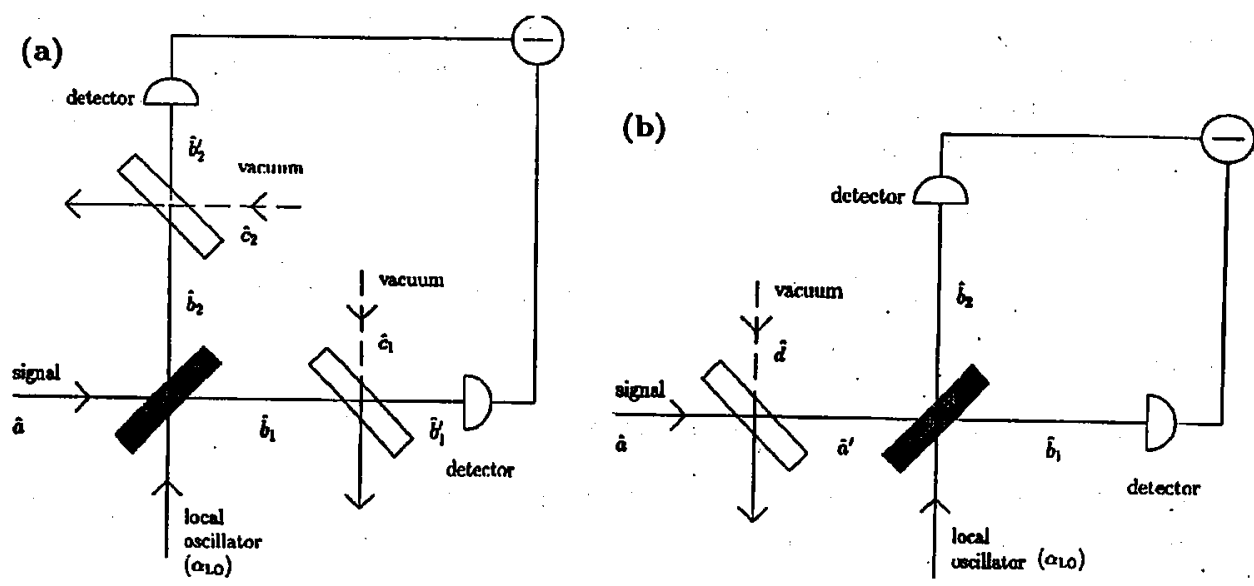

Fig. 4. (a) Balanced homodyne detection scheme with nonideal detectors modelled as unit-efficiency detcctors with beam splitters placed in front of them, (b) equivalent model. Indicated are the photon annihilation operators associated with the different light modes involved.

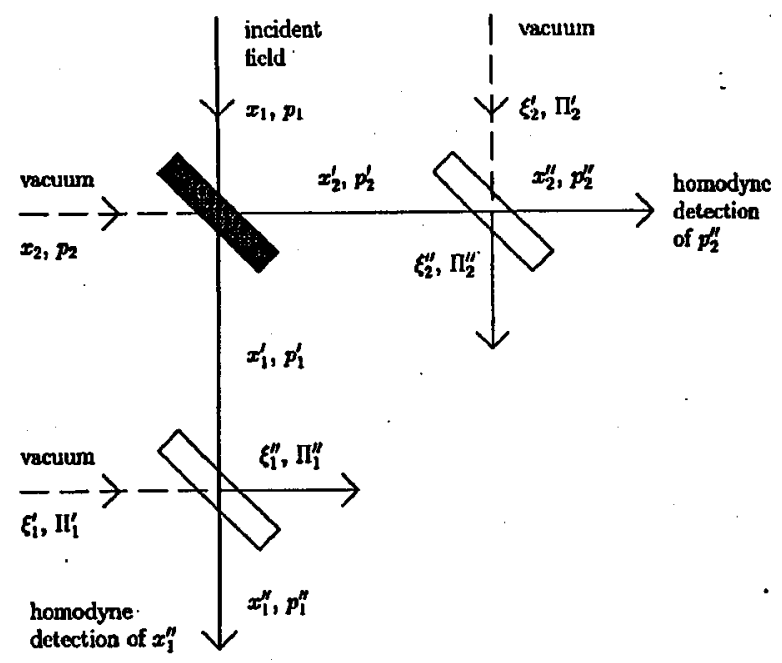

Fig. 5. Beam splitting followed by balanced homodyne detection with nonideal detectors in the equivalent model of Fig. $4 \mathrm{~b}$. Indicated are the variables in the phase spaces associated with the different light modes involved.

While for $\eta=1$ the right-hand side of Eq. (10) is, apart from a factor of 2, just the $Q$ function for the initial field $Q_{1}\left(2^{-\frac{1}{2}} x_{1}^{\prime \prime}, 2^{-\frac{1}{2}} p_{2}^{\prime \prime}\right)$ in accordance with Eq. (8), the Wigner function for the initial field $P_{1}(x, p)$ becomes more strongly smoothed for $\eta<1$, which is just what one expects. It is interesting to note that integrals of the 
type (10) - the convolution of the Wigner function with a Gaussian - are special cases of the so-called $s$-parametrized quasiprobability distributions $W(x, p ; s)$ introduced by Cahill and Glauber [13]. Quite generally, those distributions are connected through the following relation*:

$$
\begin{aligned}
& W\left(x^{\prime}, p^{\prime} ; s\right)=\pi^{-1}(t-s)^{-1} \int W(x, p ; t) \\
& \quad \times \exp \left\{-(t-s)^{-1}\left[\left(x^{\prime}-x\right)^{2}+\left(p^{\prime}-p\right)^{2}\right]\right\} \mathrm{d} x \mathrm{~d} p,(s<t) .
\end{aligned}
$$

Specializing to $t=0$ we obtain from Eq. (11):

$$
W\left(x^{\prime}, p^{\prime} ; s\right)=-\pi^{-1} s^{-1} \int P(x, p) \exp \left\{\left[\left(x^{\prime}-x\right)^{2}+\left(p^{\prime}-p\right)^{2}\right] / s\right\} \mathrm{d} x \mathrm{~d} p,
$$

where $P(x, p)=W(x, p ; 0)$ is the Wigner function. Hence our result (10) can be rewritten in the compact form

$$
w\left(x_{1}^{\prime \prime}, p_{2}^{\prime \prime}\right)=2 \eta^{-1} W_{1}\left(2^{\frac{1}{2}} \eta^{-\frac{1}{2}} x_{1}^{\prime \prime}, 2^{\frac{1}{2}} \eta^{-\frac{1}{2}} p_{2}^{\prime \prime} ;-(2-\eta) / \eta\right)
$$

where $W_{1}(x, p ; s)$ denotes the $s$-parametrized quasiprobability distribution for the initial field.

\subsection{Amplification}

In the amplification scheme [3] we have to calculate the Wigner function for the (strongly) amplified field. First we calculate the Wigner function for the whole field emerging from the amplifier which consists of a signal and an idler wave. This is done by replacing the arguments in the product of the Wigner function of the incident signal field, $P_{1}\left(x_{1}, p_{1}\right)$, and the Wigner function (3) of the vacuum noise being initially present in the idler mode, in the following way:

$$
\left(\begin{array}{l}
x_{1} \\
x_{2}
\end{array}\right)=\left(\begin{array}{cc}
C & -S \\
-S & C
\end{array}\right)\left(\begin{array}{l}
x_{1}^{\prime} \\
x_{2}^{\prime}
\end{array}\right), \quad\left(\begin{array}{l}
p_{1} \\
p_{2}
\end{array}\right)=\left(\begin{array}{ll}
C & S \\
S & C
\end{array}\right)\left(\begin{array}{l}
p_{1}^{\prime} \\
p_{2}^{\prime}
\end{array}\right)
$$

IIere, the following abbreviations have been introduced:

$$
S=\sinh \kappa T, \quad C=\cosh \kappa T,
$$

where $\kappa$ denotes the effective coupling constant and $T$ - the interaction time.

The Wigner function for the amplified signal field is then readily found by tracing over the variables for the unobserved idler, $x_{2}^{\prime}$ and $p_{2}^{\prime}$,

$$
\begin{aligned}
& P_{\text {ampl }}\left(x_{1}^{\prime}, p_{1}^{\prime}\right)=\pi^{-1} S^{-2} \int P_{1}\left(x_{1}, p_{1}\right) \\
& \quad \times \exp \left\{-C^{2} S^{-2}\left[\left(x_{1}-x_{1}^{\prime} / C\right)^{2}+\left(p_{1}-p_{1}^{\prime} / C\right)^{2}\right]\right\} \mathrm{d} x_{1} \mathrm{~d} p_{1}
\end{aligned}
$$

or, according to Eq. (12),

$$
P_{\text {ampl }}\left(x_{1}^{\prime}, p_{1}^{\prime}\right)=C^{-2} W_{1}\left(x_{1}^{\prime} / C, p_{1}^{\prime} / C ;-C^{2} / S^{2}\right) .
$$

The amplified signal field will be taken as the input for the detection scheme studied in Sec. 4.2 (see Fig. 5). Then we need only to make use of the former result

"See Eq. (6.32) in Ref. [13]. Our formula (23) differs from that equation in that $x$ and $p$ instead of $\operatorname{Re} \alpha\left(=2^{-\frac{1}{2}} x\right)$ and $\operatorname{Im} \alpha\left(=2^{-\frac{1}{2}} p\right)$ were chosen as arguments of the quasiprobability distributions. 
(10) in order to obtain the measured distribution function $w\left(x_{1}^{\prime \prime}, p_{2}^{\prime \prime}\right)$. Insertion of the result (17) into Eq. (10) and substituting $x_{1}^{\prime} / C=x, p_{1}^{\prime} / C=p$ gives us

$$
\begin{aligned}
& w\left(x_{1}^{\prime \prime}, p_{2}^{\prime \prime}\right)=2 \pi^{-1}(2-\eta)^{-1} \int W_{1}\left(x, p ;-C^{2} / S^{2}\right) \\
& \quad \times \exp \left\{-\frac{\eta C^{2}}{2-\eta}\left[\left(x-2^{\frac{1}{2}} \eta^{-\frac{1}{2}} x_{1}^{\prime \prime} / C\right)^{2}+\left(p-2^{\frac{1}{2}} \eta^{-\frac{1}{2}} p_{1}^{\prime \prime} / C\right)^{2}\right]\right\} \mathrm{d} x \mathrm{~d} p .
\end{aligned}
$$

Utilizing the general formula (11), we arrive at the final result

$$
\begin{aligned}
& w\left(x_{1}^{\prime \prime}, p_{2}^{\prime \prime}\right)=2 \eta^{-1} C^{-2} \\
& \quad \times W_{1}\left[2^{\frac{1}{2}} \eta^{-\frac{1}{2}} x_{1}^{\prime \prime} / C, 2^{\frac{1}{2}} \eta^{-\frac{1}{2}} p_{1}^{\prime \prime} / C ;-\left(1+2(1-\eta) \eta^{-1} C^{-2}\right)\right],
\end{aligned}
$$

which clearly exhibits the deteriorating effect of nonunit-efficiency detectors. It becomes evident, however, from Eq. (19) that one can tolerate low detector efficiencies, when there is strong enough amplification $(C \gg 1)$. Therefore, from the practical point of view, the amplification scheme for phase measurement is, in fact, superior to the approach based on beam splitting.

To summarize, we have demonstrated that the experimental approaches to phase measurement discussed and partly realized thus far, are physically equivalent. All of them amount to measuring the $Q$ function of the initial field from which the phase distribution follows as a marginal distribution. In the theoretical analysis, however, the unrealistic assumption was made that unit-efficiency detectors are used for the observation. In the second part of the paper the deteriorating effect of low-efficiency detectors on the measurement result was properly taken into account. It was shown that this effect gives rise to an additional smoothing process. What proves to be actually measurable is certain $s$-parametrized quasi-probability distributions instead of the $Q$ function.

\section{References}

[1] See the classic review by P. Carruthers, M.M. Nieto, Rev. Mod. Phys. 40, 111 (1968).

[2] D.T. Pegg, S.M. Barnett, Europhys. Lett. 6, 483 (1988); S.M. Barnett, D.T. Pegg, J. Mod. Opt. 36, 7 (1989).

[3] A. Bandilla, I. Paul, Ann. Phys. (Leipzig) 23, 323 (1969); H. Paul, Fortschr. Phys. 22, 657 (1974). Experiments along these lines were performed by H. Gerhardt, U. Büchler, G. Litfin, Phys. Lett. A 49, 119 (1974).

[4] J.H. Shapiro, S.S. Wagner, IEEE J. Quantum Electron. QE-20, 803 (1984).

[5] J.W. Noh, A. Fougères, L. Mandel, Phys. Rev. Lett. 67, 1426 (1991); Phys. Rev. A 45, 424 (1992).

[6] N.G. Walker, J.E. Carroll, Opt. Quantum Electron. 18, 355 (1986).

[7] A different treatment was given by M. Freyberger, K. Vogel, W. Schleich, Quantum Opt. 5, 65 (1993).

[8] The case of a wave function has been treated by U. Leonhardt, H. Paul, Phys. Rev. $A$ 47, R2460 (1993).

[9] U. Leonhardt, Phys. Rev. A 48, 3265 (1993).

[10] W. Schleich, A. Bandilla, H. Paul, Phys. Rev. A 45, 6652 (1992). 
[11] U. Leonhardt; H. Paul, Phys. Rev. A 48, 4598 (1993).

[12] H.P. Yuen, J.H. Shapiro, in: Coherence and Quantum Optics IV, Eds. L. Mandel, E. Wolf, Plenum, New York 1978, p. 719; IEEE Trans. Inf. Theory 26, 78 (1980). [13] K.E. Cahill, R.J. Glauber, Phys. Rev. 177, 1882 (1969). 